\title{
Thermal Study on Phase Transitions of Block Copolymers by Mesoscopic Simulation
}

\author{
César Soto-Figueroa ${ }^{1}$, Luis Vicente ${ }^{2}$ \\ and María del Rosario Rodríguez-Hidalgo ${ }^{1, *}$ \\ ${ }^{1}$ Departamento de Ciencias Quimicas, \\ Facultad de Estudios Superiores Cuautitlán, \\ Universidad Nacional Autónoma de México (UNAM) \\ ${ }^{2}$ Departamento de Física y Química Teórica, \\ Facultad de Química Universidad Nacional Autónoma de México (UNAM) \\ México
}

\section{Introduction}

The block copolymers are an exceptional kind of macromolecules constituted by two or more blocks of different homopolymer chains linked by covalent bonds. These polymeric materials have received much attention over past few years due in large part to their ability to selfassemble in the melted state or in a selective solvent inside a variety of ordered phases or welldefined structures of high regularity in size and shape with characteristic dimensions between 100 and 500 nanometres. These ordered phases and their structural modification are the key to many valuable physical properties which make block copolymers of great industrial and technological interest. The molecular self-assemble and formation of periodic phases in the block copolymers depend of the strength of interblock repulsion and composition, for example, mesoscopic studies of the poly(styrene)-poly(isoprene) (PS-PI) diblock copolymer, have demonstrated that this synthetic material, may generate a series of long-range ordered microdomains when exist a weak repulsion between the unlike monomers isoprene and styrene, as result, the PS-PI diblock copolymer chains tend to segregate below some critical temperature, but, as they are linked by covalent bonds, the phase separation on a macroscopic level is prevented, only a local microphase segregation occurs (Soto-Figueroa et al., 2005, SotoFigueroa et al., 2007). The phase transition from homogeneous state of polymeric chains to an ordered state with periodic phases is called microphase separation transition (MST) or orderdisorder phase transition (ODT) (Leibler, 1980). The phase segregation and generation of ordered structures in the microscopic level of a diblock copolymer via an order-disorder phase transition is illustrated in Fig. 1.

As result of microphase segregation process, the block copolymers can display ordered structures constituted by homopolymer domains that haves only mesoscopic dimensions corresponding to the size of singles blocks. The microphase separation leads to different classes of well-defined periodic structures in dependence on the ratio between the degrees of

${ }^{*}$ Corresponding Authors 


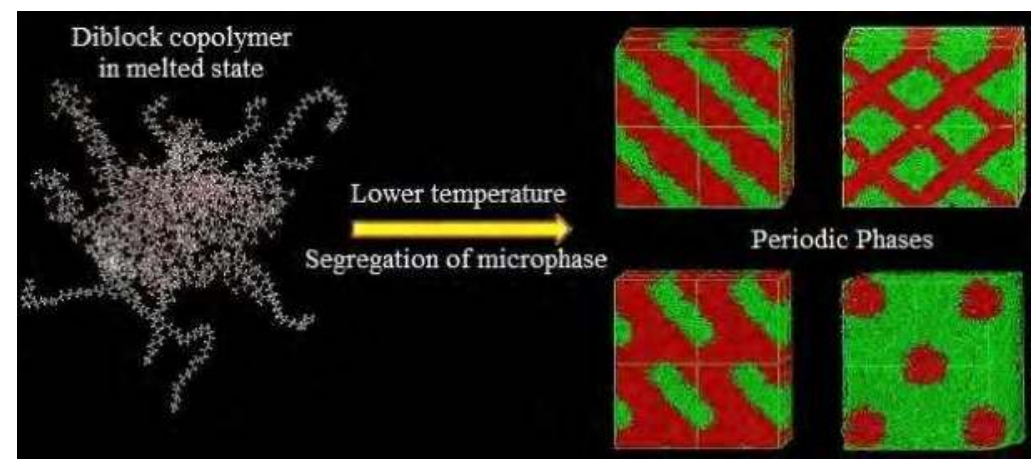

Fig. 1. The microphase segregation process occurs when the PS-PI diblock copolymers in melted state are transformed to a periodic inhomogeneous phase of ordered structures when the temperature diminishes.

polymerization of the component blocks. Periodic phases with specific morphologies such as: spherical, perforated layers, cylindrical, lamellar and Gyroid can be generated manipulating the composition or length of the component blocks (Strobl, 1997; Bates \& Fredrickson., 1990).

The order-disorder and order-order phase transitions play an important role in the design and modification of new supramolecular materials and are the key to manipulate the physical and mechanicals properties in these polymeric materials.

In this chapter, attention has been concentrated on the order-disorder and order-order phase transitions that display the PS-PI diblock copolymers and in the mesoscopic simulations methods employed to explore the kinetics transformation pathway of well-defined ordered phases.

\subsection{Order-disorder phase transition (ODT)}

The order-disorder phase transition is a thermodynamic process controlled by enthalpic and entropic interactions, for example, when a diblock copolymer of type A-B are in a melted state by temperature effect, exhibits a homogeneous phase where all different block segments are completely miscible, in the reverse case when the different block segments are immiscible due to decrease of the temperature displays an heterogeneous state of ordered microphases: this conditions are describes by Gibbs free energy equation of mixing when $\Delta \mathrm{Gm}<0$ and $\Delta \mathrm{Gm}>0$ respectively.

$$
\Delta \mathrm{G}_{\mathrm{m}}=\mathrm{G}_{\mathrm{AB}}-\left(\mathrm{G}_{\mathrm{A}}+\mathrm{G}_{\mathrm{B}}\right)
$$

where $G_{A}, G_{B}$ and $G_{A B}$, denote the Gibbs free energy of $A$ and $B$ segments in separate states and the mixed state, respectively. Equation (1) in accordance with Flory-Huggins theory can be expressed also as a sum of two thermodynamic contributions (Flory, 1953):

$$
\Delta \mathrm{G}_{\mathrm{m}}=-\mathrm{T} \Delta \mathrm{S}_{\mathrm{m}}+\Delta \mathrm{H}_{\mathrm{m}}
$$

In this equation, $\Delta \mathrm{H}_{\mathrm{m}}$ and $\mathrm{T} \Delta \mathrm{S}_{\mathrm{m}}$ exhibits the enthalpic and entropic interactions of mixing at temperature $\mathrm{T}$. The entropic and enthalpic interactions of mixing of two component segments of diblock copolymer are given by: 


$$
\begin{gathered}
\frac{\Delta \mathrm{S}_{\mathrm{m}}}{\overline{\mathrm{R}}}=\overline{\mathrm{n}}_{\mathrm{A}} \ln \phi_{\mathrm{A}}+\overline{\mathrm{n}}_{\mathrm{B}} \ln \phi_{\mathrm{B}} \\
\Delta \mathrm{H}_{\mathrm{m}}=\overline{\mathrm{R}} \mathrm{T} \frac{\mathrm{V}}{\overline{\bar{v}}_{\mathrm{c}}} \chi \phi_{\mathrm{A}} \phi_{\mathrm{B}}
\end{gathered}
$$

The equations 3 and 4 can be rewritten as:

$$
\begin{gathered}
\Delta S_{\mathrm{m}}=-\mathrm{K}\left[\mathrm{n}_{\mathrm{A}} \ln \phi_{\mathrm{A}}-\mathrm{n}_{\mathrm{B}} \ln \phi_{\mathrm{B}}\right] \\
\Delta \mathrm{H}_{\mathrm{m}}=\mathrm{kT} \chi N \phi_{\mathrm{A}} \phi_{\mathrm{B}}
\end{gathered}
$$

where $\phi_{\mathrm{A}}$ and $\phi_{\mathrm{B}}$ are volume fraction of $\mathrm{A}$ and B components of diblock copolymer, $\mathrm{N}=\mathrm{n}_{\mathrm{A}}+\mathrm{n}_{\mathrm{B}}$ denotes the total number of molecules or degree of polymerization and $\mathrm{X}$ is the Flory-Huggins interaction parameter. The phase behaviour that exhibits the diblock copolymers during an order-disorder phase transition (microphase segregation) is controlled by both entropic and enthalpic interactions. The enthalpic interactions imply the repulsion magnitude between different species via Flory-Huggin's interaction parameter $(\mathrm{X})$, which represent the chemical incompatibility between different repetitive units and its magnitude is expressed by the type of monomers which integrate the diblock copolymer and has a strong dependence with the temperature:

$$
\chi \approx \frac{1}{\mathrm{~T}}
$$

the phase segregation behaviour is controlled by the value of $x$, in this way, positives values of interaction parameter lead to incompatibility between different segments and the entropic interactions $\left(\Delta \mathrm{S}_{\mathrm{m}}\right)$ appears to be mostly positives, this generates a positive heat of mixing and therefore a $\Delta \mathrm{G}_{\mathrm{m}}>0$. Negative values of $\chi$ lead to homogeneous state and therefore a $\Delta \mathrm{G}_{\mathrm{m}}<0$.

Whereas the entropic interactions involve the configurational and translation displacement of polymeric chains, and are regulated through the degree of polymerization $N$, architecture constrains and blocks composition (Bates \& Fredrickson., 1990, 1999; Hamley, 1998; BaltaCalleja et al., 2000; Thomas et al., 1995). The microphase segregation degree in the diblock copolymers depends of the enthalpic-entropic balance represented by the reduced parameter $\chi N$, the ODT occurs at a critical value of $\chi N$, the melt phase behaviour is thus governed by composition and a reduced parameter (Leibler et al., 1980; Bates et al., 1990). Three segregation regimes have been identified by Matsen and Bates and have been defined depending on the extent of microphase segregation: the weak $(\chi N \approx 10)$, intermediate $\left(\chi_{N}>12-100\right)$ and strong segregation regimes $(\chi N>100)$ (Matsen \& Bates, 1996; Bates \& Fredrickson, 1990). In the weak segregation regime, the volume fraction of one of the block varies sinusoidally about the average value generating the formation of ordered microphases, this regime is characterized by a diffuse interface between different components and is capable of to be modified by composition effect or for temperature effect (Hamley, 1998; Bates, 1991). In the intermediate segregation regime the composition profile becomes sharper generating ordered microphases with a narrow interface between blocks. The strong segregation regime due to saturation of the blocks composition contains essentially pure components; in this regime, the phase behaviour 
depends largely on the copolymer composition. Within these segregation regimes it is possible to predict and modify the phase behaviour of block copolymers given $x, N$ and the segment length (block composition).

A general description of phase behaviour that exhibits the well-defined ordered structures of the PS-PI diblock copolymer via order-disorder phase transitions have been explored by mesoscopic simulation methods. When PS-PI diblock copolymer is in melted state, the polymeric chains assume the lowest free energy configuration, if the diblock copolymer is cooled, the repulsion magnitude expressed by reduced parameter $\chi \mathrm{N}$ increases, when the value of this parameter exceeds a certain value specific $(\chi N \approx 10.5)$ for the system under consideration, well-defined periodic structures evolves in the disordered state (SotoFigueroa et al., 2005).

When the PS-PI diblock copolymer has a symmetric composition (volume fraction of both components are the same) display an ordered phase with lamellar morphology (LAM), Fig. 2(a), however, if the volume fraction of a component increases in relative to other component (asymmetric copolymer), the interface tends to become curve. In this case, the conformational entropy loss of the majority component is too high. Therefore, to gain the conformational entropy, the chains of the majority component tend to expand along the direction parallel to interface. As a result, the PS/PI interface becomes convex towards the minority component. This interface curvature effect is more pronounced when the composition of the diblock copolymer is more asymmetric, Fig. 2(b). The PS-PI diblock copolymers with asymmetric compositions can generate a wide range of ordered structures such as the body-centred-cubic (BCC), hexagonal packed cylinders (HPC), ordered bicontinuous double diamond (OBDD or Gyroid) and lamellar (LAM) arrangement via an order-disorder phase transition process, Fig. 2(c-f).

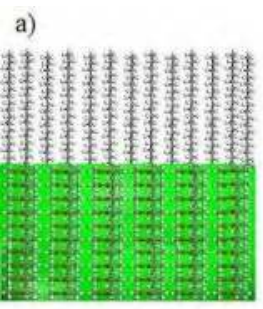

Flat interface of PS/PI

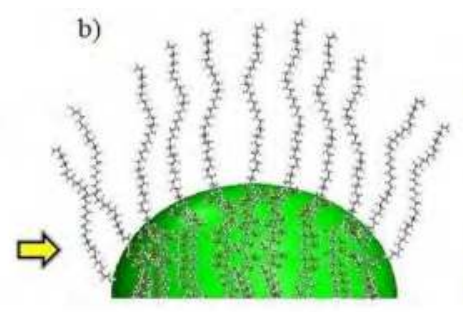

Curved interface

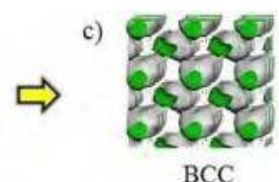

d)
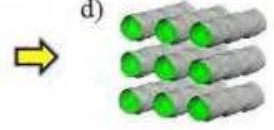

HPC

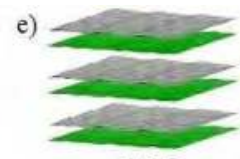

LAM

f)

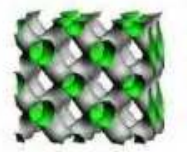

Gyroid

Fig. 2. Schematic representation of composition effect in the phase behaviour of the PS-PI diblock copolymer: a) lamellar phase (symmetric copolymer), b) curvature of PS/PI interface by effect composition (asymmetric copolymer), c) spherical phase, d) cylindrical phase, e) lamellar phase and f) OBDD phase.

The ordered phases with well-defined morphologies that shows the block copolymers are usually describes by the volume fraction of one block $(f)$, the overall degree of polymerization $(\mathrm{N})$, and Flory-Huggins interaction parameter $(\mathrm{X})$ (Soto- Figueroa et al., 2007). The periodic phases of type BCC, HPC, Gyroid and LAM explored by means of mesoscopic simulations are described in the following sections. 


\subsubsection{Body centred cubic phase}

The body centred cubic (BCC) phase or spherical phase is a classic structure of great thermodynamic stability that exhibits the diblock copolymers of type A-B. This ordered structure shows two specific symmetries: a four-fold symmetry ([100] projection) and the hexagonal symmetry ([111] projection) of cubic array respectively, see Fig. 3.

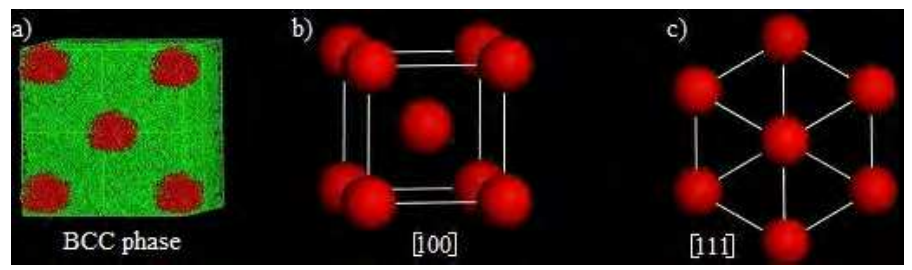

Fig. 3. Snapshots of BCC phase of PS-PI copolymer: a) ordered microdomains of PS and PI chains (red and green regions represent the PS and PI microdomain respectively), b-c) projections [100] and [111] of poly(styrene) microdomains (the poly(isoprene) matrix has been removed for a better visualization).

The BCC phase of diblock copolymers have been studied extensively by both theoretical and experimental methods. For example, the theoretical studies of PS-PI diblock copolymer with linear architecture have demonstrated that this periodic phase can be generated in a composition interval of 0.1 to 0.19 (volume fraction of poly(styrene)) with a stable four-fold symmetry (Soto-Figueroa et al., 2005). The spherical phase of this diblock copolymer is known as a stable phase, however, can be transformed on other ordered structure via an order-order phase transition.

\subsubsection{Hexagonal packed cylinders phase}

The hexagonally packed cylinders (HPC) phase is also considered as a classic phase of great thermodynamic stability due to its high packing. This ordered structure is characteristic of PS-PI diblock copolymers with linear architecture and can be generated via an orderdisorder phase transition in a composition interval of 0.2 to 0.26 (volume fraction of poly(styrene), see Fig. 4.

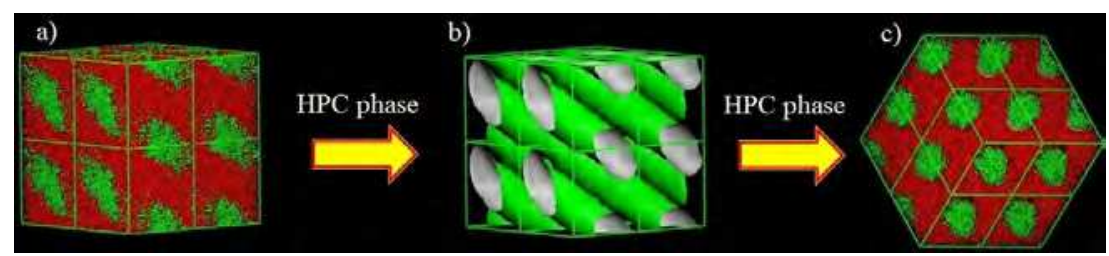

Fig. 4. Snapshots of HPC phase of PS-PI diblock copolymer: a) cylindrical microphase constituted by ordered microdomains of poly(styrene) and poly(isoprene), b) representation of density isosurfaces of microdomains of poly(styrene) and c) poly(styrene) cylinders arranged in a hexagonal lattice.

The HPC phase displays a tetrahedral arrangement of epitaxially cylinders arranged in a hexagonal lattice. In this ordered phase, the cylindrical microdomains of poly(styrene) 
exhibits an hexagonal arrangement of undulated microdomains immersed in a poly(isoprene) matrix.

\subsubsection{Ordered bicontinuous double diamond phase}

The ordered bicontinuous double diamond (OBDD) phase (or Gyroid phase) is the ordered structures more complex that exhibits some diblock copolymers of type A-B. For example, The PS-PI diblock copolymer can generate this ordered structure via an order-disorder phase transition, see Fig. 5.

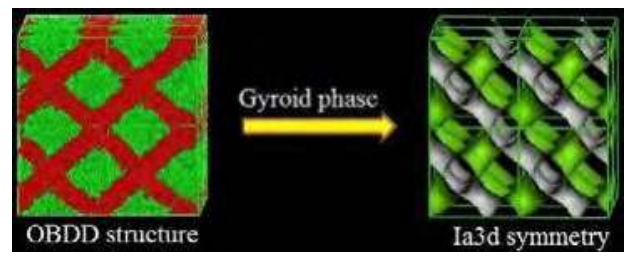

Fig. 5. Snapshots of OBDD arrangement of PS-PI diblock copolymer obtained by mesoscopic simulations: a) ordered microdomains of poly(styrene) and poly(isoprene) chains, and b) representation of density isosurfaces of microdomains of poly(styrene) (Soto-Figueroa et al., 2008).

The OBDD phase has a tetrahedral arrangement of epitaxially cylinders interconnected by channels of Ia3d symmetry (Hajduk et al., 1995). The OBDD phase of PS-PI copolymer exists only in a narrow interval of values ( 0.37 to 0.4 volume fraction of poly(styrene)) between the regimes of the perforated layer and lamellar phases. The Gyroid arrangement is known as a stable phase, however, can be transformed on other ordered structure via an order-order phase transition.

\subsubsection{Lamellar phase}

Ordered phase with lamellar morphology are characteristics of block copolymers of symmetric and asymmetric composition. This periodic phase is constituted of alternating layers of different homopolymer microdomains separates by flat interfaces, see Fig 6. For the case of PS-PI diblock copolymer with linear architecture, this displays the lamellar arrangement in a specific predominance interval between 0.47 to 0.68 volume fraction of poly(styrene).

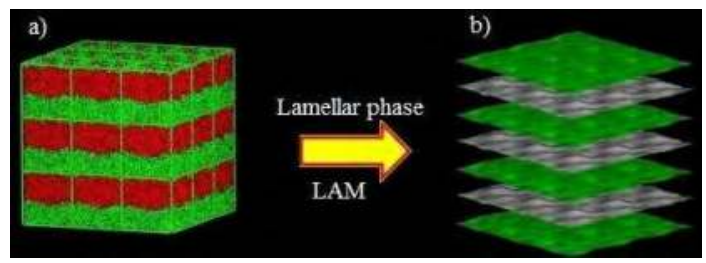

Fig. 6. Snapshots of lamellar phase of PS-PI diblock copolymer, the lamellar structure exhibits undulated microdomains of poly(styrene) and poly(isoprene): a) ordered microdomains of poly(styrene) and poly(isoprene) and b) representation of density isosurfaces of LAM phase. 
The lamellar phase can be generated in the three segregation regimes $(\chi N \approx 10, \chi N>12-100$ and $\chi N>100$ ) via order-disorder phase transitions and can displays flat and undulated interfaces.

\subsubsection{Phase diagram of PS-PI diblock copolymer}

The ordered structures of PS-PI diblock copolymer represent under specific conditions the states with the lowest Gibbs free energy, these equilibrium phases can be classified through a phase diagram, Fig. 7. The diagram phase depicts the two regions associates constituted by a homogeneous state and an ordered state of five ordered phases different. Each equilibrium phase shows its predominance zone in terms of the volume fraction of poly(styrene) block and reduced parameter $\chi \mathrm{N}$.

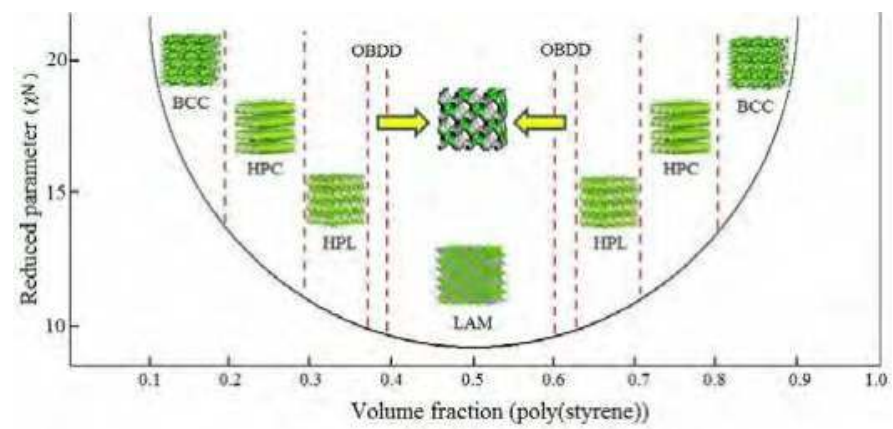

Fig. 7. Phase diagram of PS-PI diblock copolymer, the continuous curve describes the points of phase transition between the homogeneous state and the microphase-separated states. The ordered states split into different classes (BCC, HPC, G, HPL and LAM); the dashed lines show the predominance interval between the different types of ordered phases.

The segregation state in the phases diagram is controlled by the product $\chi N$, if $\chi N$ is mayor than a critical value (typically of the order of 10.5), entropic factors dominate and the diblock copolymers exist in an ordered phases state. On the other hand, an order-todisorder transition takes place for large values of $\chi \mathrm{N}$. The phase behaviour of each ordered structures kind on the phase diagram of poly(styrene)-poly(isoprene) diblock copolymer can be modified through order-order phase transitions, this will be tackled in the following section.

\section{Order-order phase transition in block copolymers}

The diblock copolymers display a wide variety of classic phases with morphologies or defined structures such as: body centred cubic, hexagonal packed cylinders, hexagonal perforated layers, lamellar and the ordered bicontinuous double diamond phase, the morphology of these periodic structures can be controlled and modified by two different routes: 1) order-disorder phase transition and 2) thermotropic order-order phase transition.

In the order-disorder phase transition as was mentioned in the previous section, the phase behaviour in the block copolymers is governed by three experimentally controllable factors during the synthesis process: i) the overall degree of polymerization, ii) architecture of block 
copolymer, and iii) the interaction parameter between components blocks, of this thermodynamic process can emerge periodic structures with well-defined morphologies (Soto-Figueroa et al., 2005, Soto-Figueroa et al. 2007).

Other way to modify the phase behaviour in the block copolymers is through the temperature; thermally induced phase transitions have the potential to promote the kinetic control in these synthetic materials. Leibler was the first in predicting the phase transition between different ordered structures by temperature effect (Leibler et al., 1980). The thermally induced phase transformations are governed for anisotropic composition fluctuation effects (Ryu et al., 1999), when an ordered phase of specific morphology is subject to a thermal heating process, the homopolymer chains into the ordered microdomains exhibit thermodynamic instability by temperature effect and become less rigid, this entropic process promotes the polymeric chains movement (composition fluctuations) into the homopolymer microdomains and modifies with the time the shape of ordered phase.

Three segregation regimes have been defined to explain the extent of microphase segregation and the thermodynamic stability in the classical phases that exhibits the diblock copolymers, these segregation regimes are : weak regime $(\chi N \approx 10)$, intermediate regime $(\chi N$ $>10-100)$ and strong segregation regime $(\chi N>100)$ (section 1.1).

Our interest is concentrated in the weak segregation regime, because in this predominance zone, the diblock copolymers are characterized by a widened interface due to enhanced phase mixing. In the vicinity to this regime, thermotropic phase transition between different kinds of ordered phases can be generating (Bates et al., 1990; Matsen et al., 1996).

The transition from one ordered state to another is nowadays denominated as an orderorder phase transition (OOT) (Sakurai et al., 1993; Kim et al., 1998; Almadal et al., 1992; Sakurai et al., 1996; Sakamato et al., 1997; Modi et al., 1999). In this way, the ordered phases that exhibit the diblock copolymers inside weak segregation regime can exhibit order-order phase transitions when are subjects to thermal heating cycles.

In the past decade the order-order phase transitions that display the classical phases of diblock copolymers (BCC, HPC, Gyroid and Lamellar) have been investigated by both experimental and theoretical studies. The order-order phase transition is thermoreversible process that develops transient metastable states during phase transformation. The orderorder phase transition that exhibits the classical phases with specific morphology such as Gyroid, cylindrical, and lamellar are described next.

\subsection{Order-order phase transition of HPC structure}

The ordered phase of hexagonal packed cylinders also known as cylindrical structure is characteristic of diblock and triblock copolymers, for example, the poly(styrene)poly(isoprene) diblock copolymer with linear architecture can generate this ordered arrangement in a specific composition of 0.2/0.8 (volume fraction ) of PS/PI via an orderdisorder phase transition. When the HPC phase of this diblock copolymer is subject to thermal heating cycles, exhibits an order-order phase transition to body-centred-cubic (BCC) structure (or spherical phase). The order-order transition pathway that exhibits the HPC to BCC phase was recently reported (Modi et al., 1999; Krishnamoorti et al., 2000). 
Experimental and theoretical studies have confirmed that HPC phase develops transient metastable phases during thermal heating process (Kimishima et al., 2000; Krishnamoorti et al., 2000; Rodríguez-Hidalgo et al., 2009). The order-order transition pathway from HPC to spherical phase is sketched schematically in Fig. 8.

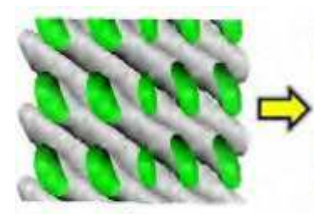

a)

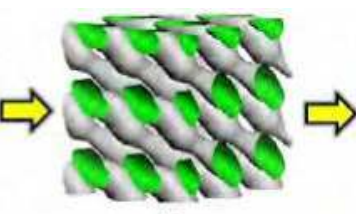

b)

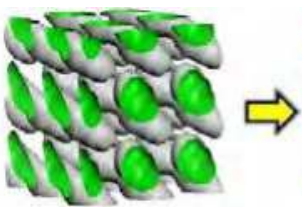

c)

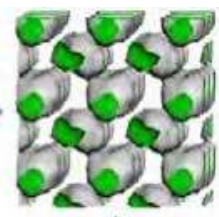

d)

Fig. 8. Snapshots of order-order phase transition process from HPC phase to spherical arrangement monitored by means of mesoscopic simulations: a) cylindrical phase, $b$ ) undulation process of poly(styrene) microdomains by temperature effects, c) breakdown of cylindrical microdomains of poly(styrene) and d) formation and stabilization of bodycentred-cubic arrangement (BCC phase) (Soto-Figueroa et al., 2008).

Three transitory stages are typical of OOT process from HPC to cylindrical phase: (Stage 1) undulation process of cylindrical microdomains of poly(styrene) in the poly(isoprene) matrix. In this transitory stage the thermal heating induce the thermodynamic instability of HPC phase, where the cylindrical microdomains and the PS/PI interface becomes less rigid due to anisotropic composition fluctuations, this generates the undulation of the poly(styrene) microdomain in the poly(isoprene) matrix. (Stage 2) breakdown of cylindrical microdomains of poly(styrene) by temperature effects. When anisotropic composition fluctuations reach a critical point of thermodynamic instability the cylindrical microdomains become unstable and therefore they are broken in ellipsoids, (Stage 3) formation and stabilization of BCC phase. In this thermally induced stage, the ellipsoids microdomains generated by breakdown of cylindrical microdomains of poly(styrene) evolve to an equilibrium state where the uniform spherical microdomains are stabilized into bodycentred-cubic arrangement. The order-order phase transition from HPC to spherical phase is a thermoreversible process. The inverse process from BCC state to HPC arrangement involves deformation and elongation of spheres into ellipsoids and coalescence of ellipsoids into the cylindrical microdomains. This process is driven by thermodynamic instability of the spherical interface caused by decrease of temperature.

\subsection{Order-order phase transition of Gyroid structure}

The Gyroid phase as was mentioned in the section 1.1.3 exhibits a tetrahedral arrangement of epitaxially cylinders interconnected by channels of Ia3d symmetry and is capable of suffering order-order phase transitions when it is submitted to thermal heating cycles. The poly(styrene)-poly(isoprene) diblock copolymer displays this ordered phase in a narrow interval of specific composition. The thermally-induced phase transition from Gyroid to lamellar phase in PS-PI diblock copolymer was explored in detail by mesoscopic simulation methods (Soto-Figueroa et al., 2008). The phase transformation during thermal annealed proceed in several stages via the generation two metastable phases (HPL and cylindrical). The order-order phase transition process from Gyroid to lamellar phase is sketched schematically in Fig. 9. 


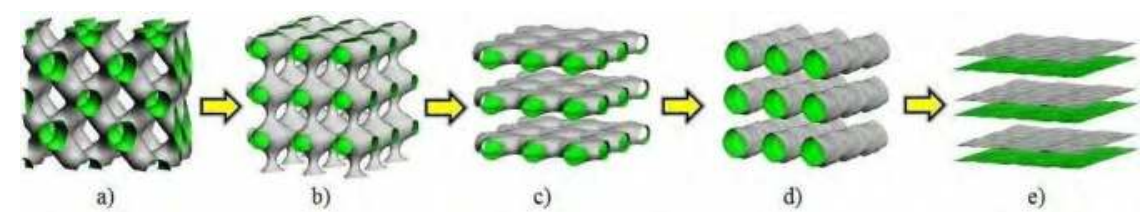

Fig. 9. Transitory stages and metastable phases generated during the order-order phase transition from Gyroid phase to lamellar arrangement: a) undulation of interconnected microdomains of poly(styrene) into the poly(isoprene) matrix, due thermal heating, $b$ ) breakdown of interconnected microdomains of Gyroid phase, c) formation of HPL metastable phase, d) formation of cylindrical metastable phase and e) lamellar phase.

When the Gyroid phase of PS-PI copolymer is subject to thermal heating cycles, the interconnected microdomains of poly(styrene) display an undulation process due to anisotropic composition fluctuations, Fig 9(a), with the increase of temperature the anisotropic composition fluctuations induce the breakdown of side interconnections in the Gyroid arrangement, Fig. 9(b), generating the first metastable phase with HPL arrangement, Fig. 9(c). The HPL metastable phase evolves later to cylindrical arrangement by temperature effect, in this transient stage the interconnections of perforated layer microdomains diminish their volume up to the breakdown of interconnections in the HPL arrangement and the formation of cylindrical phase (second metastable phase), Fig 9(d). Finally, the cylindrical metastable phase also changes over time due to anisotropic composition fluctuations. The cylindrical microdomains in this metastable phase are thermodynamically unstable, in order to reach a thermodynamic stability of minimal energy, the cylinders microdomains are joined together to evolve into undulating lamellar phase, Fig. 9(e), the lamellar phase consisting of alternating layers of PS and PI microdomains.

\subsection{Order-order phase transition of lamellar structure}

The lamellar phase is the ordered structure more simple that exhibit the multiblock copolymers is considered a classical phase of great thermodynamic stability. The lamellar phase consisting of alternating layers of different homopolymer microdomains. The PS-PS diblock copolymer exhibits this ordered arrangement in a specific composition interval (see section 1.1.4). When the lamellar phase of this diblock copolymer with an equivalent composition between their constituent blocks ( 0.5 volume fraction of poly(styrene) and poly(isoprene)) is subject to thermal heating cycles, it does not generate an order-order phase transition, the lamellar arrangement in this case evolve to a homogeneous state (melted), see Fig. 10.

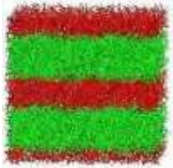

a)

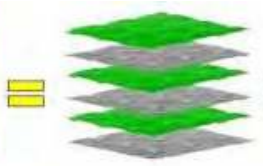

b)
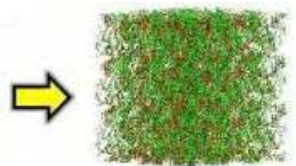

c)

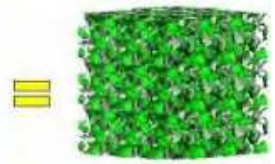

d)

Fig. 10. Snapshots of thermal heating of lamellar phase (diblock copolymer of symmetric composition): $a-b$ ) equilibrium phase of lamellar structure (representation of diblock chains and surface isodensities of PS and PI microdomains) and c-d) homogeneous state after of thermal heating (melted phase). 
Nevertheless, the lamellar phase generated of block copolymers of asymmetric composition, for example of $0.45 / 0.55$ of PS/PI (volume fraction), it can exhibit a phase transformation to HPL arrangement when is subject to thermal heating via an order-order phase transition process (Mani et al., 2000). The order-order phase transition from lamellar to HPL phase is showed in Fig. 11.

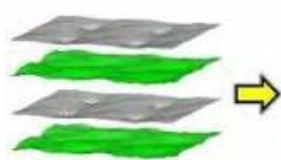

a)

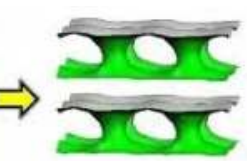

b)

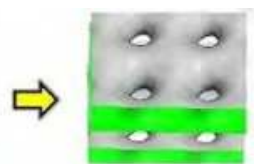

c)

Fig. 11. Order-order phase transition stage from lamellar phase to HPL arrangement: a) undulation process of lamellar microdomains, $b$ ) interconnection of parallel microdomains of poly(styrene) and c) formation and stabilization of HPL arrangement.

The order-order phase transition process of this ordered arrangement is governed by the thermodynamic instability between the components microdomains, generated by thermal heating, where the anisotropic composition fluctuations play an important role in the phase transformation. In the initial stage the lamellar microdomains exhibit an undulation process due to thermal heating, Fig. 11(a), with the temperature increase, the lamellar phase becomes thermodynamically unstable, in order to reach a thermodynamic stability of minimal energy, the lamellar alternate microdomains of poly(styrene) are interconnected by means of narrow microdomains (parallels to PS/PI interface), finally the HPL phase evolve in an energetic equilibrium state (Soto-Figueroa et al., 2007), see Fig. 11(b-c).

\section{Thermal study of double directionality of order-order phase transitions of hexagonally perforated layers (HPL) phases by mesoscopic simulation}

During the past two decades have been reported theoretical and experimental studies of diblock copolymers, where the ODT and OOT transitions play a main role in the structure control and consequently in the physical properties control of these polymeric materials (Kimishima et al., 2000; Kim et al., 2006; Bodycomb et al., 2000; Krishanamoorti et al., 2000; Court et al., 2006; Soto-Figueroa et al., 2008). It is well-known that the poly(styrene)poly(isoprene) diblock copolymer exhibits a wide variety of classical phases with specific morphologies such as: BCC, HPC, OBDD, LAM and HPL. The order-order phase transitions that exhibit this ordered structures with definite morphologies have been explored by SotoFigueroa and Rodríguez-Hidalgo, they have confirmed the OOT's between HPC to BCC, Gyroid to LAM microphase through mesoscopic simulations (Soto-Figueroa et al., 2007, 2008; Rodríguez-Hidalgo et al., 2009), although the OOT's have been well investigated for the majority of classical phases, the dynamic transformation of HPL phase has not been investigated yet in detail. The HPL phase exhibits a double directionality of order-order phase transition when is subject to thermal process. Experimental evidences suggest that HPL phase of the PS-PI copolymer can evolve to a cylindrical structure and to a Gyroid structure by temperature effect (Park et al., 2005; You et al., 2007).

The double directionality of order-order phase transitions that exhibits the classical phase of PS-PI copolymer is a topic interesting to control the morphology and physical 
properties of polymeric materials. The order-order phase transitions can be investigated in more detail through mesoscopic simulations than through experimentation. Mesoscopic simulations are efficient methods to investigate the physical processes of soft matter and their interactions with chemical environments (Rodríguez-Hidalgo et al., 2011; Ramos-Rodriguez et al., 2010). Offer a particularly useful way of exploring the matter transfer process and to make predictions that may be of interest for understanding and elucidating complex process such as the double directionality of order-order phase transitions. In the mesoscopic simulations the atoms of each molecule are not directly represented, but they are grouped together into beads (coarse-grained models), where a springs force reproduce the typical nature of them, and therefore can exhibit a real physical behaviour in multicomponent systems. In this section, we explored the double directionality of phase transition of HPL structure of the PS-PI copolymer from a mesoscopic point of view by mesoscopic simulations, where the phase evolution stages and transient ordered states are analysed.

\subsection{Model and simulation method}

To explore the order-order phase transition of the HPL phase, we employed Dissipative Particle Dynamics (DPD) simulations. The original DPD method was introduced by Hoogerbrugge and Koelman and was later modified by Groot, R.D. (Hoogerbrugge \& Koelman, 1992; Koelman \& Hoogerbrugge, 1993; Groot \& Warren, 1997; Groot \& Madden, 1998, 1999). The DPD method allows the study of high-molecular-weight systems as the polymeric materials. The coarse-graine

$$
f_{i}=m_{i} \frac{d v_{i}}{d t}
$$

where $\mathbf{r}_{i}, \mathbf{v}_{i}, \mathrm{~m}_{\mathrm{i}}$ and $\mathbf{f}_{i}$ are the position, velocity, mass, and force, respectively, of bead $i$. Dimensionless units are used in DPD simulations; usually, the mass of each bead is set to 1 DPD mass units, which results in an equation between the force acting on a bead and its acceleration. Each particle is subject to soft interactions with its neighbours via three forces: conservative $\left(\mathrm{F}_{\mathrm{ij}}^{\mathrm{C}}\right)$, dissipative $\left(\mathrm{F}_{\mathrm{ij}}^{\mathrm{D}}\right)$ and random $\left(\mathrm{F}_{\mathrm{ij}}^{\mathrm{R}}\right)$. The total force acting on particle $i$ is:

$$
\mathrm{f}_{i}=\sum_{\mathrm{j} \neq \mathrm{i}}\left(\mathrm{F}_{\mathrm{ij}}^{\mathrm{C}}+\mathrm{F}_{\mathrm{ij}}^{\mathrm{D}}+\mathrm{F}_{\mathrm{ij}}^{\mathrm{R}}\right)
$$

The conservative force $\mathrm{F}_{\mathrm{ij}}^{\mathrm{C}}$ is a soft repulsive force that acts between particles $i$ and $j$. The dissipative force $\mathrm{F}_{\mathrm{ij}} \mathrm{D}$ corresponds to a frictional force that depends on both the positions and relative velocities of the particles. The random $f \mathbf{v}_{i}=\frac{r_{i}}{d t}$ orce $F_{i j}{ }^{R}$ is a random interaction between a bead $i$ and its neighbour bead $j$. All forces vanish beyond a cut-off radius $r_{c}$, which is usually chosen as the reduced unit of length, $r_{c} \equiv 1$. The $F_{i j}{ }^{D}$ and $F_{i j}{ }^{R}$ forces act as a thermostat that conserves momentum and gives the correct hydrodynamics at sufficiently large time and length scales. These forces are given by: 


$$
\begin{gathered}
F_{i j}^{C}=\left[\begin{array}{ll}
a_{i j}\left(1-r_{i j}\right) \hat{r}_{i j} & \left(r_{i j} \leq 1\right) \\
0 & \left(r_{i j}>1\right)
\end{array}\right] \\
F_{i j}^{D}=\left[-\gamma \omega^{D}\left(r_{i j}\right)\left(\mathbf{v}_{i j} \hat{\mathbf{r}}_{i j}\right) \hat{r}_{i j}\right] \\
F_{i j}^{R}=\left[\sigma \omega^{R}\left(r_{i j}\right) \xi_{i j} \hat{\mathbf{r}}_{i j}\right]
\end{gathered}
$$

where $r_{i j}=\left|\vec{r}_{i}-\vec{r}_{j}\right|, \hat{r}=\vec{r}_{i j} / r_{i j}, \gamma$ is the dissipation strength, $\sigma$ is the noise strength, $\omega^{D}\left(r_{i j}\right)$ and $\omega^{R}\left(r_{i j}\right)$ are weight functions of $F_{i j}{ }^{D}$ and $F_{i j}{ }^{R}$ forces, respectively, and $a_{i j}$ is the maximum repulsive force between particle $i$ and $j$. For the DPD system to have a well-defined equilibrium state that obeys Boltzmann statistics, the equilibrium temperature is defined as $k_{B} T=\sigma^{2} /(2 \gamma)$. This condition fixes the temperature of the system and relates with the two DPD parameters $\gamma$ and $\sigma\left(\mathrm{k}_{\mathrm{B}} \mathrm{T}\right.$ is usually chosen as the reduced unity of energy). The parameter $\mathrm{a}_{\mathrm{ij}}$ (henceforth referred to as the bead-bead repulsion parameter or simply as the DPD interaction parameter) depends on the underlying atomistic interactions and is related to the parameter $\chi$ through:

$$
\mathrm{a}_{\mathrm{ij}}=\mathrm{a}_{\mathrm{ii}}+\frac{\mathrm{k}_{\mathrm{B}} \mathrm{T} \chi_{\mathrm{ij}}(\mathrm{T})}{0.306}
$$

In this way, a connection exists between the molecular character of the coarse-grained model and the DPD parameter. The parameter $\mathrm{a}_{\mathrm{ij}}$ is given in terms of $\mathrm{k}_{\mathrm{B}} \mathrm{T}$ (DPD reduced units). Equation (6) implies that, if the species are compatible, $\chi_{\mathrm{ij}} \approx 0$ and therefore, $\mathrm{a}_{\mathrm{ij}}=25$. Established procedures for mapping between the DPD and physical scales and for choosing the system temperature are not yet available. We therefore use Flory-Huggins theory (through the $\chi(\mathrm{T})$ dependence) to introduce the temperature into the DPD simulations. To calculate the $\mathrm{a}_{\mathrm{ij}}$ values of Equation (6), we use the Hildebrand relation:

$$
X=\frac{V_{m}}{R T}\left(\delta_{1}-\delta_{2}\right)^{2}
$$

where $V_{m}$ and $\delta$ are the mean molar volume and solubility parameter, respectively. In the DPD simulation, the dynamic behaviour of order-order phase transition of the HPL structure is followed by integration of the equations of motion of each species using a modified version of the Verlet algorithm (Verlet, 1967). The integration of the equations of motion for each particle generates a trajectory through the system's phase, from which thermodynamic observables may be constructed by suitable averaging. Based on this information, the orderorder phase transition can be observed. In this algorithm, the forces are still updated once per integration, thus there is virtually no increase in computational cost.

\subsection{Coarse-grained models and parameterization}

The molecular structure of PS-PI copolymer was built by means of the polymer builder module of Accelrys (Accelrys, 2006). The polymeric molecule with linear architecture 
contains a total of 300 repetitive units in the main chain. The molecular weight of the PS-PI copolymer presented an interval of $20798-25842 \mathrm{~g} / \mathrm{mol}$. The diblock copolymer molecule was replaced by a coarse-grained model constituted by 30 beads, where each bead represents a statistical segment (characteristic ratio, $\left(\mathrm{C}_{\mathrm{n}} \approx 10\right)$ (Soto-Figueroa, et al 2005)), see Figure 12. The Equation (15) was used to map the real structure of diblock copolymer to statistical model (mesoscopic model).

$$
\mathrm{C}_{\mathrm{SGD}}=\frac{\mathrm{M}_{\mathrm{P}}}{\mathrm{M}_{\mathrm{m}}(\mathrm{SSL})}
$$

where $\mathrm{C}_{\mathrm{SGD}}, \mathrm{M}_{\mathrm{P}}, \mathrm{M}_{\mathrm{m}}$ and SSL are bead number with Gaussian distribution, the molar mass of the block copolymer, molar mass of a repeat unit and means statistical segment level (characteristic ratio $\left(C_{n}\right)$, or persistence length $\left(L_{p}\right)$ or statistical Kuhn segment length $\left(a_{k}\right)$ ) respectively (Soto-Figueroa et al., 2007).

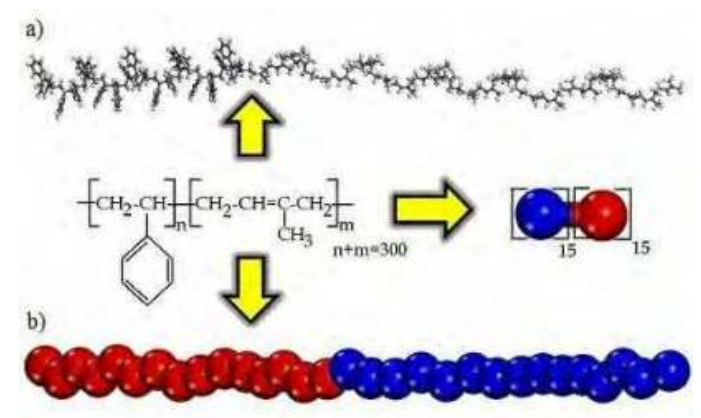

Fig. 12. Schematic representation of PS-PI copolymer: a) chemical structure of a PS-PI chain, $\mathrm{n}$ and $\mathrm{m}$ represent the polymeric unit number of each block, $\mathrm{b}$ ) coarse-grained model of PSPI system with linear architecture.

The chemical and physical nature of the coarse-grained model in mesoscopic simulation is described by interaction parameters $\left(\chi_{\mathrm{ij}}\right)$. In order to study the order-order transition at temperatures different with DPD simulations we take the temperature dependence $\chi_{\mathrm{ij}}(T)=$ $\chi(T)$, in this way the real temperature is introduced into the DPD simulation (RodríguezHidalgo et al., 2009). The interaction parameters for Equation (13) were evaluated from bulk atomistic simulations using the Fan, F.C. model (Fan et al., 1992). In this way the parameter interaction was expressed as:

$$
\chi(T)=\frac{\Delta G(T)}{R T}=\frac{Z_{12} E_{12}(T)+Z_{21} E_{21}(T)-Z_{11} E_{11}(T)-Z_{22} E_{22}(T)}{2 R T}
$$

where $\Delta G$ denotes the Gibbs free energy, $\chi$ is interaction parameter, $Z$ and $\Delta E_{12}$ are coordination number and differential energy of interaction of an unlike pair respectively. The Figure 13 show the interaction parameters $\chi(T)$, the temperature interval analysed is from $298 \mathrm{~K}$ to $500 \mathrm{~K}$.

The tendencies that exhibit interaction parameters in temperature function are in agreement with the Hildebrand relation and are adequate to explore the formation of HPL phases (via 


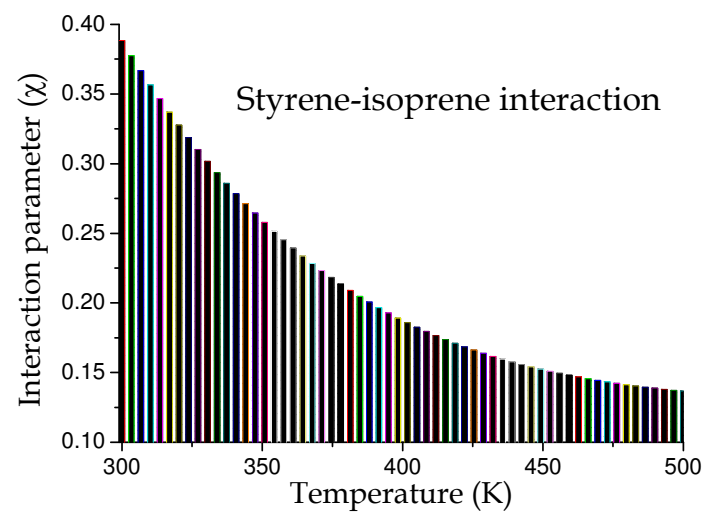

Fig. 13. Interaction parameters between styrene and isoprene molecules at temperatures different obtained by Monte Carlo molecular simulation (Vicente et al. 2006).

order-disorder transition) to room temperature and the double directionality of the HPL phase (via order-order transition) employing the coarse-grained model with predefined architecture proposed in this mesoscopic study.

All DPD simulations were performed in a cubic box that measured $20 \times 20 \times 20$ in size, which contained a total of $2.4 \times 10^{4}$ representatives particles, a spring constant of $\mathrm{C}=4$, and a density of $\rho=3$. The interaction parameters between identical species were then chosen as $\mathbf{a}_{\text {ST-ST }}=\mathbf{a}$ PI$\mathrm{PI}=25$. Each bead was assigned a radius of 1 . The coarse-grained number for each chemical species was held constant during the DPD simulations.

\subsection{Simulation results and discussion}

\subsubsection{Hexagonally perforated layers structures}

The equilibrium phases formation of PS-PI copolymer is governed by the composition, temperature and immiscibility between their components blocks, these factors were considered in the coarse-grained model via the architecture and interaction parameters.

All simulations start from a disordered state, where the PS-PI chains are in a homogeneous melted phase. First, we set the interactions parameters at $\mathrm{T}=298 \mathrm{~K}$. We then let the simulation proceed for $5 \times 10^{5}$ steps and during the temperature relaxation; we observed the microphase segregation process and the generation of equilibrium phases via ODT. Several transient stages were detected in the ODT process; a) melt phase, where the copolymer chains move freely; b) microphase segregation by temperature effect (temperature decrease), c) generation of pure microdomains of poly(styrene) and poly(isoprene), in this stage the ordered phases system attains an equilibrium temperature to room temperature.

To identify the composition region where the HPL structures are formed, we scanned the composition interval from 0.1-0.5 (volume fraction of poly(styrene) with increments of 0.03 ) at constant temperature of $298 \mathrm{~K}$ (i.e. constant $\chi \mathrm{N}$ ). All DPD simulations generate a coarsegrained system sufficiently large to observe the classical phases formation in the analysed composition interval. The mesoscopic simulation of PS-PI copolymer exhibits a wide variety of structures or equilibrium phases such as LAM, HPC, BCC, Gyroid and HPL. The 
equilibrium phases of PS-PI system depend primarily on three factors: (i) the volume fraction of PS and PI blocks ( $f$ ), (ii) the degree of polymerization $(N)$, and (iii) the interaction parameter $(\chi)$. The results obtained by DPD simulations are in accordance with the meanfield theory (Leibler, 1980).

The HPL phase was obtained into the composition intervals of 0.3 to 0.36 (volume fraction of poly(styrene)), see Figure 14. The tendency of PS-PI chains to self-assemble into HPL structures depends of the previous factors and is governed by thermodynamics interactions (enthalpic and entropic) during the microphase segregation process.

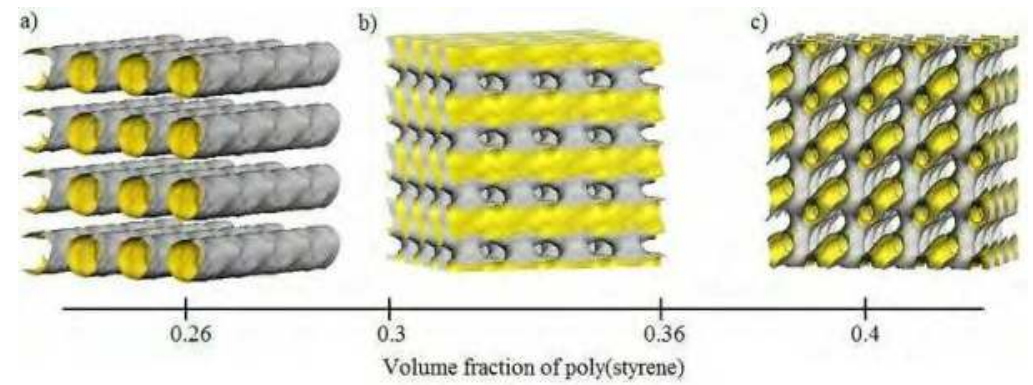

Fig. 14. Specific phases of PS-PI copolymer obtained as the blocks composition (PS/PI) is modified: a) cylindrical, b) HPL and c) Gyroid.

The thermodynamic stability of HPL phase in the predominance region vary with the relative chain length (poly(styrene) composition) of the component blocks. The predominance region where the HPL phase is formed has two composition limits that are contiguous with other equilibrium phases of different morphology. In the low composition limit where the volume fraction of poly(styrene) is close to 0.3, the HPL structure has as neighbour the cylindrical phase, whereas in the height composition limit (close to 0.36), the HPL structure has as neighbour the Gyroid structure. These behaviours between different phases are in accordance with the phase diagram reported by Khandpur (Khandpur et al., 1995).

The order-order phase transitions that exhibits the HPL structures with specific compositions of 0.3 and 0.36 (volume fraction of poly(styrene) are investigated. The HPL structures are modified through temperature increase, keeping constant the PS-PI block composition. The OOT of HPL structure for each specific composition shows a selective directionality (from HPL to cylinders and from HPL to Gyroid) during thermal study.

\subsubsection{Order-order phase transition of HPL to cylindrical phase}

The HPL phase with a specific composition of 0.3 (volume fraction of poly(styrene) was put at continuous cycles of thermal heating in the temperature interval from 298 to $500 \mathrm{~K}$. A total of $2.0 \times 10^{5}$ time steps with step size of $\Delta t=0.03$ were allowed in the mesoscopic simulation to reach the thermodynamic balance of PS-PI system in each temperature increment. The thermally induced phase transition from HPL to cylindrical phase was observed at the temperature of $432 \mathrm{~K}$, this corresponds to the OOT, and this fact is in accordance with theoretical and experimental evidences (You et al., 2007). 
The OOT process was visualized during mesoscopic simulation; the results show that the transformation from HPL to cylindrical phase by temperature effect is generated in several stages. When the HPL phase is annealed below the OOT temperature, we observed a slow dynamic motion of the PS and PI microdomains, the thermodynamic stability between microdomains different they are in an energetic barrier that maintain the HPL phase stable.

When the HPL phase is annealed to a higher temperature, $\mathrm{T} \geq 432 \mathrm{~K}$, the energetic barrier that maintain the HPL phase stable is exceeded, the thermodynamic interactions (enthalpic and entropic) play an important role in the OOT process. The enthalpic interaction is proportional to the Flory-Huggins interaction parameter (Fig. 13), which is found to be inversely proportional to temperature.

During the temperature increase, the interaction parameter between the PS and PI microdomains diminishes, generating the OOT from HPL arrangement to cylindrical phase, the enthalpic interaction in the OOT is accompanied by an increase in entropy. At higher temperatures the entropic interactions dominates and is cause of anisotropic composition fluctuations into polymeric microdomains (Ryu, et al., 1999).

The HPL phase develops short-lived transient states during the OOT process, because of interface instability (PS/PI), combined with fast molecular motion of PS and PI microdomains. In Figure 15(a-d) are shown snapshots of the thermally induced phase transition from HPL to cylindrical structure during the annealed process at temperature, $\mathrm{T}=432 \mathrm{~K}$.

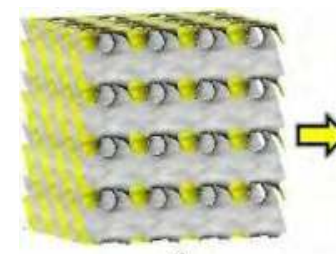

a)

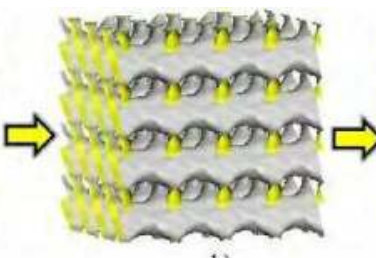

b)

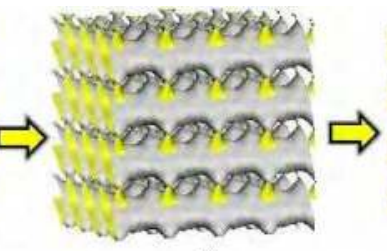

c)

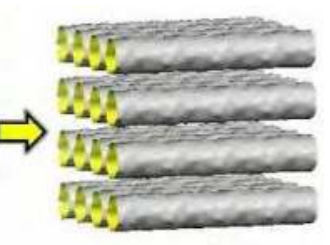

d)

Fig. 15. Snapshots of the OOT pathway from HPL to cylindrical phase: a) undulation process of HPL phase, b-c) instability of HPL phase and d) formation of cylindrical phase.

The snapshot of Figure 15(a) corresponds to the initial equilibrium state of HPL phase, initially, the PS perforated microdomains display an undulation process induced by thermodynamic instability, in this point the PS perforated microdomains and the PS/PI interface become less rigid by temperature effect. The PS microdomains maintain their shape an short-period of time, as time goes, the anisotropic composition fluctuations (thermally-induced) increase quickly, the PI interconnections into PS perforated microdomains enlarge their area, in this stage the PS microdomains are unstable see Figure $15(b-c)$. When the anisotropic composition fluctuations reach a critical point, the PS unstable microdomains change their structure to a new cylindrical arrangement, see Figure 15(d), in this thermally induced stage the uniform cylindrical microdomains are stabilized.

\subsubsection{Order-order phase transition of HPL to Gyroid phase}

The HPL phase with a volume fraction of PS, $\mathrm{f}_{\mathrm{PS}}=0.36$, (obtained within the first $5 \times 10^{5}$ time steps) was now subject to a thermal heating process for another $5 \times 10^{5}$ time steps. The 
simulation outcome shows an OOT from HPL to Gyroid phase at the temperature of $438 \mathrm{~K}$. The composition increase of poly(styrene) in HPL microdomains modify the phase transition directionality. The OOT from HPL to Gyroid phase obtained by DPD simulation are consistent with experimental results reported by Insun Park. They have investigated the phase transition behaviour from the hexagonally perforated layer (HPL) to the Gyroid phase in supported thin film of a poly(styrene)- $b$-poly(isoprene) (PS- $b$-PI) diblock copolymer (OOT occur at temperature of $443 \mathrm{~K}$ ) (Park et al., 2005).

During the thermal heating process, the HPL phase undergoes the microdomains modification of PS and PI, generating transient intermediate stages as are sketched schematically in Figure 16(a-c). Three transient intermediate stages were detected during the OOT: (i) undulation process of pure microdomains and PS/PI interface due to the thermodynamic instability by temperature effect, see Figure 16(a), in this stage, the entropic and enthalpic interactions govern the microphase stability and induce anisotropic composition fluctuations into pure microdomains, (ii) increase of volume of poly(isoprene) interconnections into PS perforates microdomain, Figure 16(b), in this stage the PS perforated microdomains are instable, (iii) formation of parallel interconnections between PS perforated layers, see Figure 16(c), in this stage, the Gyroid phase is formed.

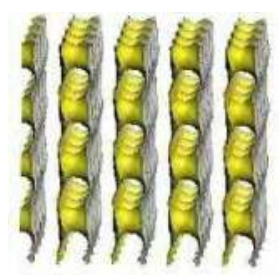

a)
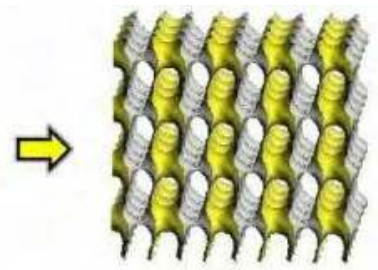

b)

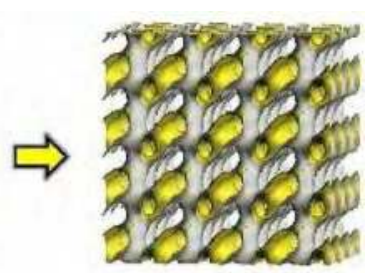

c)

Fig. 16. Snapshots of evolution process of the OOT from HPL to Gyroid phase: a) undulation process of PS microdomains in the PI matrix by anisotropic composition fluctuations effect, b) volume increase of PI interconnections into PS perforates microdomains, c) formation of Gyroid phase by generation of parallel interconnections between PS perforated microdomains.

The HPL phase of PS-PI diblock copolymer exhibits a double directionality of order-order transition from HPL to cylindrical phase and from HPL to Gyroid phase. The anisotropic composition fluctuations of PS and PI microdomains of HPL phase by thermal heating process induce the order-order phase transitions, however the phase transition directionality towards a specific phase (cylindrical or Gyroid) in the thermal process is governed by small variations of poly(styrene) concentration in the poly(styrene) microdomains of HPL phase. Variations of small composition into structured microdomains according to Leibler's mean-field theory greatly modify the phase thermodynamic behaviour (separation, segregation and phase transformation).

\section{Conclusion}

The block copolymers are "smart" materials that have the ability to self-assemble inside a variety of periodic phases of high regularity in size and shape. The phase behaviour (type of structure and morphology) that exhibits these polymeric materials can be controlled by two 
different processes: order-disorder phase transition and thermotropic order-order phase transition. In the order-disorder phase transition, the phase behaviour is governed by entropic and enthalpic interactions, whereas the order-order phase transition are controlled by anisotropic composition fluctuations of theses thermodynamic processes can emerge supramolecular structures with well-defined morphologies and specific properties.

The ODT and OOT that exhibits of block copolymer can be explored in more detail through mesoscopic simulations than through experimentation. The mesoscopic simulation methods, offer a particularly useful way of exploring the phase behaviour pathway and to make predictions that may be of interest for understanding and elucidating complex process such as order-disorder of order-order phase transitions.

The DPD approach has been successfully applied to the investigation of phase transition processes (ODT and OOT) of HPL structure. The mesoscopic simulation outcomes show that the HPL phase of PS-PI diblock copolymer exhibits a double directionality of orderorder phase transition from HPL to cylindrical phase and from HPL to Gyroid phase. This double directionality of OOT is controlled by small variations of poly(styrene) concentration in the poly(styrene) microdomains of HPL phase. Finally, all the simulation outcomes are qualitatively consistent with the experimental results, demonstrating that the DPD method may provide a powerful tool for the investigation and analysis of soft matter transformation process by thermal heating effects.

\section{Acknowledgment}

This work was supported by the Universidad Nacional Autónoma de México (UNAM), PAPIIT project No. IN104410-2 and IN109712. We also acknowledges the financial support of the Consejo Nacional de Ciencia y Tecnología (CONACYT) Project: 2012.

\section{References}

Almdal, K.; Koppi, K.A.; Bates, F.S.; Mortensen, K. (1992). Multiple ordered phases in a block copolymer melt. Macromolecules, Vol. 25(6), pp. 1743-1751.

Accelrys. (2006), Material studio release, notes, release 5.0; Accelrys Software, Inc.: San Diego, CA.

Bates, F.S. \& Fredrickson, G.H. (1990). Block copolymer thermodynamics: Theory and experiment. Annu. Rev. Phys. Chem., Vol. 41, pp. 525-557.

Bates, F.S. \& Fredrickson, G.H. (1999), Block copolymers-Designer soft materials. AIP Phys. Today, 2, 32-38.

Bates, F. S. (1991). Polymer - Polymer phase behavior. Science, Vol. 251, pp. 898.

Bodycomb, J.; Yamaguchi, D.; Hashimoto, T. (2000). A Small-Angle X-ray Scattering Study of the Phase Behavior of Diblock Copolymer/Homopolymer Blends. Macromolecules, Vol. 33(14), pp. 5187-5197.

Balta-Calleja, F.J. \& Roslaniec, Z. (2000), Block Copolymers; Marcel-Dekker Publishers, (Eds.): New York.

Court, F.; Yamaguchi, D.; Hashimoto, T. (2006). Morphological Studies of Binary Mixtures of Block Copolymers: Temperature Dependence of Cosurfactant Effects. Macromolecules, Vol. 39(7), pp. 2596-2605. 
Fan, F.C.; Olafson, B.D.; Blanco, M. (1992). Application of molecular simulation to derive phase diagrams of binary mixtures. Macromolecules, Vol. 25, pp. 3667-3676.

Flory, P. J. (1953). Principles of Polymer Chemistry, Cornell University Press, Ithaca, New York,

Groot, R. D. \& Warren, P. B. (1997). Dissipative particle dynamics: bridging the gap between atomistic and mesoscopic simulation. J. Chem. Phys., Vol. 107, pp. 4423-4435.

Groot, R. D. \& Madden, T. J. (1998). Dynamic simulation of diblock copolymer microphase separation. J. Chem. Phys., Vol. 108, pp. 8713-8724.

Groot, R. D. \& Madden, T. J., Tildesley, D. J. (1999). On the role of hydrodynamic interactions in block copolymer microphase separation. J. Chem. Phys., Vol. 110, pp.9739-9749.

Hoogerbrugge, P. J. \& Koelman, J. M. V. A. (1992). Simulating microscopic hydrodynamic phenomena with Dissipative particle dinamics. Europhys. Lett.,Vol. 19, pp.155-160.

Hajduk, D. A.; Harper, P.E.; Gruner, S. M.; Honeker, C. C.; Thomas, E. L.; Fetters, L. J. (1995). A Reevaluation of Bicontinuous Cubic Phases in Starblock Copolymers. Macromolecules, Vol. 28, pp. 2570-2573.

Hamley, I.W. (1998). The Physics of Block Copolymers. Oxford University Press, ISBN 0198502184.

Koelman, J. M. V. A. \& Hoogerbrugge, P. J.(1993). Dynamic simulation of hard sphere sispension under steady shear. Europhys. Lett., Vol. 21, 363-368.

Kimishima, K.; Koga, T.; Hashimoto, T. (2000). Order-Order Phase Transition between Spherical and Cylindrical Microdomain Structures of Block Copolymer. I. Mechanism of the Transition. Macromolecules, Vol. 33(3), pp. 968-977.

Kim, E.Y.; Lee, D.J.; Kim, J.K. (2006). Phase Behavior of a Binary Mixture of a Block Copolymer with Lower Disorder-to-Order Transition and a Homopolymer. Macromolecules, Vol. 39(25), pp. 8747-8757.

Kim, J.K.; Lee, H.H.; Gu, Q.J.; Chang, T.; Jeong, Y.H. (1998). Determination of Order-Order and Order-Disorder Transition Temperatures of SIS Block Copolymers by Differential Scanning Calorimetry and Rheology. Macromolecules, Vol. 31(12), pp. 4045-4048.

Krishanamoorti, R.; Modi M.A.; Tse, M.F.; Wang H.-C. (2000). Pathway and Kinetics of Cylinder-to-Sphere Order-Order Transition in Block Copolymers. Macromolecules, Vol. 33, pp. 3810-3817.

Krishanamoorti, R.; Silva A. S.; Modi M.A. (2000). Small-Angle Neutron Scattering Study of a Cylinder to Sphere order-order Transition in Block Copolymers. Macromolecules, Vol. 33, pp. 3803-3809.

Khandpur, A.K.; Forster, S. ; Bates, F.S.; Hamley, I.W.; Ryan, A.J.; Bras, W.; Almadal, K.; Mortensen, K. (1995). Polyisoprene-Polystyrene Diblock Copolymer Phase Diagram near the Order-Disorder Transition. Macromolecules, Vol 28(26), pp. 8796-8806.

Leibler, L. (1980). Theory of Microphase Separation in Block Copolymers. Macromolecules, Vol. 13(6), pp. 1602-1607.

Matsen, M. W. \& Bates, F. S. (1996). Unifying Weak- and Strong-Segregation Block Copolymer Theories. Macromolecules, Vol. 29, pp. 1091-1098.

Modi, M.A.; Krishanamoorti, R.; Tse, M.F.; Wang, H.C. (1999). Viscoelastic Characterization of an Order-Order Transition in a Mixture of Di- and Triblock Copolymers. Macromolecules, Vol. 32(12), pp. 4088-4097. 
Mani, S.; Weiss, R. A.; Cantino, M. E.; Khairallah, L. H.; Hans, S. F.; Williams, C. E. (2000). Evidence for a Thermally Reversible Order-Order Transition between Lamellar and Perforated Lamellar Microphases in a Triblock Copolymer. Eur. Polym. J., Vol. 36, pp. 215-219.

Park, I.; Lee, B.; Ryu, J.; Im, K.; Yoon, J.; Ree, M.; Chang, T. (2005). Epitaxial Phase Transition of Polystyrene-b-Polyisoprene from Hexagonally Perforated Layer to Gyroid Phase in Thin Film. Macromolecules, Vol. 38, pp.10532-10536.

Ramos-Rodriguez, D. A.; Rodriguez-Hidalgo, M. R.; Soto-Figueroa, C.; Vicente, L. (2010). Molecular and mesoscopic study of ionic liquids and their use as solvents of active agents released by polymeric vehicles. Molecular Physics, Vol. 108(5), pp. 657-665.

Rodríguez-Hidalgo, M.R.; Soto-Figueroa, C.; Vicente, L. (2011). Mesoscopic simulation of the drug release mechanism on the polymeric vehicle $\mathrm{P}(\mathrm{ST}-\mathrm{DVB})$ in an acid environment. Soft Matter, Vol. 7, pp 8224-8230.

Rodríguez-Hidalgo, M.R.; Soto-Figueroa, C.; Vicente, L. (2009). Mesoscopic study of cylindrical phases of poly(styrene)-poly(isoprene) copolymer: Order-order phase transitions by temperature control. Polymer, Vol. 50, pp 4596-4601.

Ryu, C.Y. \& Lodge, T.P. (1999). Thermodynamic Stability and Anisotropic Fluctuations in the Cylinder-to-Sphere Transition of a Block Copolymer. Macromolecules, Vol. 32, pp. 7190-7201.

Sakurai, S.; Kawada, H.; Hashimoto, T.; Fetters, L.J. (1993).Thermoreversible Morphology Transition between Spherical and Cylindrical microdomains of Block Copolymers. Macromolecules, Vol. 26(21), pp. 5796-5802.

Sakurai, S.; Hashimoto, T.; Fetters, L.J. (1996). Thermoreversible Cylinder-Sphere Transition of Polystyrene-block-polyisoprene Diblock Copolymers in Dioctyl Phthalate Solutions. Macromolecules, Vol. 29(2), pp. 740-747.

Sakamato, N.; Hashimoto, T.; Han, C.D.; Kim, D.; Vaidya, N.Y. (1997). Order-Order and Order-Disorder Transitions in a Polystyrene-block-Polyisoprene-block-Polystyrene Copolymer. Macromolecules, Vol. 30(6), pp. 1621-1632.

Soto-Figueroa, C.; Rodríguez-Hidalgo, M.R.; Martínez-Magadán, J.M. (2005). Molecular simulation of diblock copolymers; morphology and mechanical properties. Polymer, Vol. 46, pp. 7485-7493.

Soto-Figueroa, C.; Luis-Vicente.; Martínez-Magadán, J.M.; Rodríguez-Hidalgo, M.R. (2007). Self-Organization Process of Ordered Structures in Linear and Star Poly(styrene)Poly(isoprene) Block Copolymers: Gaussian Models and Mesoscopic Parameters of Polymeric Systems. J. Phys. Chem. B, Vol. 111, pp. 11756-11764.

Soto-Figueroa, C.; Luis-Vicente.; Martínez-Magadán, J.M.; Rodríguez-Hidalgo, M.R. (2007). Mesoscopic simulation of asymmetric-copolymer/homopolymer blends: Microphase morphological modification by homopolymer chains solubilization. Polymer, Vol. 48, pp. 3902-3911.

Soto-Figueroa, C.; Rodríguez-Hidalgo, M.R.; Martínez-Magadán, J.M.; Luis-Vicente. (2008). Dissipative Particle Dynamics Study of Order-Order Phase Transition of BCC, HPC, OBDD, and LAM Structures of the Poly(styrene)-Poly(isoprene) Diblock Copolymer. Macromolecules, Vol. 41, pp. 3297-3304.

Soto-Figueroa, C.; Rodríguez-Hidalgo, M.R.; Martínez-Magadán, J.M.; Luis-Vicente. (2008). Mesoscopic simulation of metastable microphases in the order-order transition 
from gyroid-to-lamellar states of PS-PI diblock copolymer. Chemical Physics Letters, Vol. 460, pp. 507-511.

Strobl, G. (1997). The Physic of Polymers. Springer, ISBN 3-540-63203-4.

Thomas, E.L. \& Lescanec, R.L. (1995). Phase morphology in block copolymer systems, In A. Keller, M. Warner and A.H. Windle (Eds.), Self-order and Form in Polymeric Materials, p.147-164. Chapman \& Hall, London.

Verlet, L. (1967). Computer "Experiments" on Classical Fluids. I. Thermodynamical Properties of Lennard- Jones Molecules. Phys. Rev., Vol.159, pp. 98-103.

Vicente, L.; Soto-Figueroa, C.; Pacheco-Sanchez, H.; Hernadez-Trujillo, J.; Martínez Magadán, J. M. (2006). Fluid Phase Equilibria. Vol. 239, pp. 100-106.

You, L. Y.; Chen, L.J.; Qian, H.J.; Lu, Z.Y. Microphase Transitions of Perforated Lamellae of Cyclic Diblock Copolymers under Steady Shear. Macromolecules, 2007, Vol. 40(14), pp. 5222-5227. 


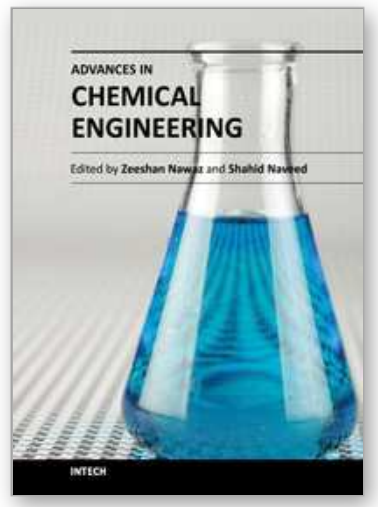

\author{
Advances in Chemical Engineering \\ Edited by Dr Zeeshan Nawaz
}

ISBN 978-953-51-0392-9

Hard cover, 584 pages

Publisher InTech

Published online 23, March, 2012

Published in print edition March, 2012

Chemical engineering applications have been a source of challenging optimization problems in terms of economics and technology. The goal of this book is to enable the reader to get instant information on fundamentals and advancements in chemical engineering. This book addresses ongoing evolutions of chemical engineering and provides overview to the sate of the art advancements. Molecular perspective is increasingly important in the refinement of kinetic and thermodynamic molding. As a result, much of the material was revised on industrial problems and their sophisticated solutions from known scientists around the world. These issues were divided in to two sections, fundamental advances and catalysis and reaction engineering. A distinct feature of this text continues to be the emphasis on molecular chemistry, reaction engineering and modeling to achieve rational and robust industrial design. Our perspective is that this background must be made available to undergraduate, graduate and professionals in an integrated manner.

\title{
How to reference
}

In order to correctly reference this scholarly work, feel free to copy and paste the following:

César Soto-Figueroa, Luis Vicente and María del Rosario Rodríguez-Hidalgo (2012). Thermal Study on Phase Transitions of Block Copolymers by Mesoscopic Simulation, Advances in Chemical Engineering, Dr Zeeshan Nawaz (Ed.), ISBN: 978-953-51-0392-9, InTech, Available from: http://www.intechopen.com/books/advancesin-chemical-engineering/thermal-study-on-phase-transitions-of-block-copolymers-by-mesoscopic-simulation

\section{INTECH}

open science | open minds

\author{
InTech Europe \\ University Campus STeP Ri \\ Slavka Krautzeka 83/A \\ 51000 Rijeka, Croatia \\ Phone: +385 (51) 770447 \\ Fax: +385 (51) 686166 \\ www.intechopen.com
}

\author{
InTech China \\ Unit 405, Office Block, Hotel Equatorial Shanghai \\ No.65, Yan An Road (West), Shanghai, 200040, China \\ 中国上海市延安西路65号上海国际贵都大饭店办公楼 405 单元 \\ Phone: +86-21-62489820 \\ Fax: $+86-21-62489821$
}


(C) 2012 The Author(s). Licensee IntechOpen. This is an open access article distributed under the terms of the Creative Commons Attribution 3.0 License, which permits unrestricted use, distribution, and reproduction in any medium, provided the original work is properly cited. 